Supporting Information

\title{
Characterization of Large Amplitude Motions and Hydrogen Bonding Interactions in the Thiophene-Water Complex by Rotational Spectroscopy
}

\author{
Weslley G. D. P. Silva and Jennifer van Wijngaarden*
}

Department of Chemistry, University of Manitoba, Winnipeg, Manitoba, R3T 2N2, Canada

${ }^{*}$ Corresponding author

Email: vanwijng@cc.umanitoba.ca

Phone: (204)474-8379

Fax: (204)474-7608

Appendix 1: Cartesian coordinates for the conformers of thiophene-w Appendix 2: Assigned transitions and residuals for the observed conformer of thiophene-w Appendix 3: Full set of calculated spectroscopic parameters for conformer I of thiophene-w 
Appendix 1: Cartesian coordinates for the conformers of thiophene-w

Table S1. Cartesian coordinates of conformer I obtained at the B2PLYP-D3(BJ)/def2-TZVP level

\begin{tabular}{cccc}
\hline \multicolumn{4}{c}{ Cartesian Coordinates (Angstroms) } \\
\hline Atom & $\mathrm{X}$ & $\mathrm{Y}$ & $\mathrm{Z}$ \\
\hline $\mathrm{C}$ & -0.925293 & 0.411849 & -1.084408 \\
$\mathrm{C}$ & -0.316248 & 1.463377 & -0.454135 \\
$\mathrm{C}$ & 0.065843 & 1.150140 & 0.878095 \\
$\mathrm{C}$ & -0.260564 & -0.134116 & 1.228478 \\
$\mathrm{~S}$ & -1.035542 & -0.958938 & -0.059518 \\
H & -1.313903 & 0.372027 & -2.088390 \\
$\mathrm{H}$ & -0.145611 & 2.420477 & -0.923510 \\
$\mathrm{H}$ & 0.557753 & 1.841239 & 1.546273 \\
$\mathrm{H}$ & -0.089015 & -0.632086 & 2.168257 \\
$\mathrm{O}$ & 2.910053 & -0.448003 & -0.283490 \\
H & 2.682679 & -0.763899 & -1.162251 \\
H & 2.076111 & -0.132049 & 0.083516 \\
\hline
\end{tabular}


Table S2. Cartesian coordinates of conformer Il obtained at the B2PLYP-D3(BJ)/def2-TZVP level

\begin{tabular}{cccc}
\hline \multicolumn{4}{c}{ Cartesian Coordinates (Angstroms) } \\
\hline Atom & $\mathrm{X}$ & $\mathrm{Y}$ & $\mathrm{Z}$ \\
\hline $\mathrm{C}$ & -1.994018 & -0.389195 & 0.000016 \\
$\mathrm{C}$ & -1.916196 & 0.977448 & 0.000012 \\
$\mathrm{C}$ & -0.574728 & 1.445202 & -0.000008 \\
$\mathrm{C}$ & 0.341110 & 0.427520 & -0.000020 \\
$\mathrm{~S}$ & -0.434202 & -1.102586 & -0.000007 \\
H & -2.876908 & -1.006386 & 0.000029 \\
H & -2.783162 & 1.621488 & 0.000024 \\
H & -0.298096 & 2.489151 & -0.000014 \\
H & 1.418347 & 0.484990 & -0.000036 \\
O & 3.724794 & 0.140015 & 0.000009 \\
H & 4.269497 & -0.067794 & 0.764643 \\
H & 4.269586 & -0.067750 & -0.764574 \\
\hline
\end{tabular}


Table S3. Cartesian coordinates of conformer III obtained at the B2PLYP-D3(BJ)/def2-TZVP level

\begin{tabular}{|c|c|c|c|}
\hline \multirow[b]{2}{*}{ Atom } & \multicolumn{3}{|c|}{ Cartesian Coordinates (Angstroms) } \\
\hline & $\mathrm{X}$ & $\mathrm{Y}$ & Z \\
\hline C & .14 & 0.81 & 0.31 \\
\hline C & 8 & - & 0. \\
\hline C & 2.080545 & -0.451 & -0.2 \\
\hline $\mathrm{C}$ & 1.619660 & 0.836813 & -0.223995 \\
\hline S & -0.044997 & 0.894796 & 0.187812 \\
\hline $\mathrm{H}$ & -1.081336 & -1.275449 & 0.562898 \\
\hline $\mathrm{H}$ & 1.226382 & -2.467236 & 0.075633 \\
\hline $\mathrm{H}$ & 3.102277 & -0.710559 & -0.492354 \\
\hline $\mathrm{H}$ & 2.16 & 1.7 & -0.411602 \\
\hline $\mathrm{O}$ & -3.280322 & -0.264850 & -0.20 \\
\hline $\mathrm{H}$ & -4.162486 & 0.113503 & -0.147055 \\
\hline $\mathrm{H}$ & -2.859895 & 0.175440 & -0.946294 \\
\hline
\end{tabular}


Table S4. Cartesian coordinates of conformer IV obtained at the B2PLYP-D3(BJ)/def2-TZVP level

\begin{tabular}{cccc}
\hline \multicolumn{4}{c}{ Cartesian Coordinates (Angstroms) } \\
\hline Atom & $\mathrm{X}$ & $\mathrm{Y}$ & $\mathrm{Z}$ \\
\hline $\mathrm{C}$ & 1.473007 & -1.120028 & -0.000018 \\
$\mathrm{C}$ & 0.116685 & -1.305486 & 0.000017 \\
$\mathrm{C}$ & -0.605758 & -0.081816 & 0.000045 \\
$\mathrm{C}$ & 0.223056 & 1.007661 & 0.000031 \\
$\mathrm{~S}$ & 1.876102 & 0.546924 & -0.000017 \\
$\mathrm{H}$ & 2.249125 & -1.867114 & -0.000044 \\
$\mathrm{H}$ & -0.348245 & -2.280279 & 0.000024 \\
$\mathrm{H}$ & -1.684165 & -0.011317 & 0.000075 \\
$\mathrm{H}$ & -0.049155 & 2.050005 & 0.000045 \\
$\mathrm{O}$ & -4.078833 & 0.142123 & -0.000018 \\
H & -4.661116 & 0.179419 & 0.764232 \\
H & -4.660949 & 0.179469 & -0.764393 \\
\hline
\end{tabular}


Appendix 2: Assigned transitions and residuals for the observed conformer of thiophene-w

Table S5. Assigned transitions and residuals for the parent species the observed conformer

\begin{tabular}{cccccccccc}
\hline$J$ & $K_{a}^{\prime}$ & $K_{\mathrm{c}}^{\prime}$ & $\mathrm{v}^{\prime}$ & $J^{\prime \prime}$ & $K_{\mathrm{a}}^{\prime \prime}$ & $K_{\mathrm{c}}^{\prime \prime}$ & $\mathrm{v}^{\prime \prime}$ & $v_{\mathrm{obs}} / \mathrm{MHz}$ & $v_{\mathrm{obs}-\mathrm{calc}}$ \\
\hline 2 & 1 & 2 & 0 & 1 & 1 & 1 & 0 & 7727.9358 & 0.0022 \\
2 & 1 & 2 & 1 & 1 & 1 & 1 & 1 & 7748.7076 & 0.0003 \\
2 & 0 & 2 & 0 & 1 & 0 & 1 & 0 & 7912.5292 & 0.0000 \\
2 & 0 & 2 & 1 & 1 & 0 & 1 & 1 & 7933.6215 & -0.0005 \\
2 & 1 & 1 & 0 & 1 & 1 & 0 & 0 & 8146.5282 & 0.0002 \\
2 & 1 & 1 & 1 & 1 & 1 & 0 & 1 & 8166.4924 & -0.0001 \\
3 & 1 & 3 & 0 & 2 & 1 & 2 & 0 & 11576.8750 & -0.0014 \\
3 & 1 & 3 & 1 & 2 & 1 & 2 & 1 & 11608.4783 & -0.0003 \\
3 & 0 & 3 & 0 & 2 & 0 & 2 & 0 & 11808.5114 & -0.0003 \\
3 & 0 & 3 & 1 & 2 & 0 & 2 & 1 & 11841.8562 & 0.0004 \\
3 & 2 & 2 & 0 & 2 & 2 & 1 & 0 & 11904.7201 & 0.0013 \\
3 & 2 & 2 & 1 & 2 & 2 & 1 & 1 & 11935.4044 & 0.0000 \\
3 & 2 & 1 & 0 & 2 & 2 & 0 & 0 & 12001.9472 & -0.0006 \\
3 & 2 & 1 & 1 & 2 & 2 & 0 & 1 & 12029.8164 & -0.0001 \\
3 & 1 & 2 & 0 & 2 & 1 & 1 & 0 & 12202.7501 & -0.0006 \\
3 & 1 & 2 & 1 & 2 & 1 & 1 & 1 & 12233.2612 & 0.0003 \\
4 & 1 & 4 & 0 & 3 & 1 & 3 & 0 & 15410.2640 & -0.0009 \\
4 & 1 & 4 & 1 & 3 & 1 & 3 & 1 & 15453.0865 & 0.0000 \\
4 & 0 & 4 & 0 & 3 & 0 & 3 & 0 & 15643.6069 & 0.0005 \\
4 & 0 & 4 & 1 & 3 & 0 & 3 & 1 & 15690.3906 & 0.0000 \\
4 & 2 & 3 & 0 & 3 & 2 & 2 & 0 & 15852.8011 & -0.0006 \\
4 & 2 & 2 & 0 & 3 & 2 & 1 & 0 & 16082.7275 & 0.0000 \\
4 & 2 & 2 & 1 & 3 & 2 & 1 & 1 & 16118.2466 & 0.0000 \\
4 & 1 & 3 & 0 & 3 & 1 & 2 & 0 & 16235.8842 & 0.0005 \\
4 & 1 & 3 & 1 & 3 & 1 & 2 & 1 & 16277.7696 & -0.0001 \\
5 & 1 & 5 & 0 & 4 & 1 & 4 & 0 & 19226.5890 & 0.0009 \\
5 & 1 & 5 & 1 & 4 & 1 & 4 & 1 & 19280.9584 & 0.0000 \\
5 & 0 & 5 & 0 & 4 & 0 & 4 & 0 & 19423.5126 & -0.0004 \\
5 & 0 & 5 & 1 & 4 & 0 & 4 & 1 & 19483.7760 & -0.0001 \\
\hline & & & & & & & & & \\
\hline
\end{tabular}


Table S6. Assigned transitions and residuals for the ${ }^{18} \mathrm{O}$ isotopic species of the observed conformer

\begin{tabular}{cccccccccc}
\hline$J^{\prime}$ & $K_{\mathrm{a}}^{\prime}$ & $K_{\mathrm{c}}^{\prime}$ & $V^{\prime}$ & $J^{\prime}$ & $K_{\mathrm{a}}^{\prime \prime}$ & $K_{\mathrm{c}}^{\prime \prime}$ & $\mathrm{v}^{\prime \prime}$ & $v_{\mathrm{obs}} / \mathrm{MHz}$ & $v_{\text {obs - calc }}$ \\
\hline 2 & 1 & 2 & 0 & 1 & 1 & 1 & 0 & 7282.2081 & 0.0078 \\
2 & 0 & 2 & 0 & 1 & 0 & 1 & 0 & 7451.3914 & -0.0018 \\
2 & 0 & 2 & 1 & 1 & 0 & 1 & 1 & 7470.8678 & 0.0065 \\
2 & 1 & 1 & 0 & 1 & 1 & 0 & 0 & 7657.0922 & -0.0060 \\
2 & 1 & 1 & 1 & 1 & 1 & 0 & 1 & 7675.9726 & -0.0065 \\
3 & 1 & 3 & 0 & 2 & 1 & 2 & 0 & 10912.0412 & -0.0011 \\
3 & 1 & 3 & 1 & 2 & 1 & 2 & 1 & 10941.0749 & -0.0011 \\
3 & 0 & 3 & 0 & 2 & 0 & 2 & 0 & 11131.8727 & -0.0043 \\
3 & 0 & 3 & 1 & 2 & 0 & 2 & 1 & 11162.1952 & -0.0021 \\
3 & 2 & 2 & 0 & 2 & 2 & 1 & 0 & 11203.3950 & 0.0009 \\
3 & 2 & 1 & 0 & 2 & 2 & 0 & 0 & 11275.9299 & -0.0035 \\
3 & 1 & 2 & 0 & 2 & 1 & 1 & 0 & 11473.1051 & 0.0080 \\
3 & 1 & 2 & 1 & 2 & 1 & 1 & 1 & 11501.7856 & 0.0032 \\
\hline
\end{tabular}


Appendix 3: Full set of calculated spectroscopic parameters for conformer I of thiophene-w

Table S7. Calculated rotational and centrifugal distortion constants for conformer I of thiophenew obtained at the B2PLYP-D3(BJ)/def2-TVZP level of theory.

\begin{tabular}{cc}
\hline Parameter & AMA-I-w-I \\
\hline$A / \mathrm{MHz}$ & 3461 \\
$B / \mathrm{MHz}$ & 1983 \\
$\mathrm{C} / \mathrm{MHz}$ & 1788 \\
$D_{\mathrm{J}} / \mathrm{kHz}$ & 8.612 \\
$D_{\mathrm{JK}} / \mathrm{kHz}$ & 5.282 \\
$D_{\mathrm{K}} / \mathrm{kHz}$ & 1.214 \\
$d_{1} / \mathrm{kHz}$ & -0.425 \\
$d_{2} / \mathrm{kHz}$ & -0.090 \\
\hline
\end{tabular}

